\title{
The Death of Micro-Organisms During Drying in Relation to Solute Concentration and Drying Temperature
}

\author{
BY R. H. LEACH*, D. F. OHYE AND W. J. SCOTT \\ Commonwealth Scientific and Industrial Research Organization, Division of Food \\ Preservation and Transport, Homebush, Nero South Wales, Australia
}

SUMMARY : A mixture of Pseudomonas fluorescens and coliphage T2 was dried at $-20,0$ and $+20^{\circ}$ in solutions of each of the 16 combinations of mannitol and sucrose at $0,0.25,0.50$, and $0.75 \mathrm{M}$. For $P$. fluorescens there was no significant average effect of the drying temperature on the numbers which survived drying, but for coliphage $T 2$ average survival increased as the drying temperature was changed from -20 to $20^{\circ}$. For both organisms, however, there were a number of very highly significant interactions between solutions and drying temperatures. In other words, the effects of the temperature depended on the solutes present and vice versa. There were also some substantial differences between the responses of the two organisms. It is concluded that survival during drying is likely to be determined by factors additional to those studied in these experiments.

The most suitable procedures for drying micro-organisms without loss of viability have not yet been defined. There is, however, a widespread opinion that drying from the frozen state gives the best results especially for organisms easily destroyed by drying. For example, Fry (1954) in reviewing results with bacteria stated that 'all the evidence shows that drying from the frozen state is desirable for all organisms and is apparently essential for the more sensitive ones'. Similarly, Harris (1954) suggested that labile viruses should be freeze-dried 'at the lowest convenient temperature'. On the other hand, very many types of micro-organisms show at least a small fraction which survives after a great variety of natural and artificial drying procedures, in many of which drying has undoubtedly occurred at temperatures substantially above $0^{\circ}$. In addition, there have been some comparisons which show that drying above $0^{\circ}$ gives greater survival than freeze-drying. Stamp (1947) found that slow drying of Serratia marcescens over $\mathrm{P}_{2} \mathrm{O}_{5}$ at room temperature gave approximately three times as many survivors as either of two methods of freeze-drying. For two types of fungal spores Mazur \& Weston (1956) found that spray-drying caused little or no mortality, whereas freeze-drying killed more than $90 \%$ of the spores. Greiff, Blumenthal, Chiga \& Pinkerton (1954) compared freeze-drying of influenza virus when the ampoules were suspended in baths at $0,-30,-60$ and $-80^{\circ}$. At $-80^{\circ}$ and at $0^{\circ}$ survival was nearly $100 \%$, but was much less at the two intermediate temperatures. In these experiments of Greiff et al. the actual temperatures of the virus suspension were not measured during drying and, as the system was evacuated, the temperature of the product might have been much lower than the temperature

* Present address: Wellcome Research Laboratories, Langley Court, Beckenham, Kent. 
of the baths in which the ampoules were immersed. We have found specimen temperatures within evacuated ampoules to be up to $60^{\circ}$ below the temperature of the bath surrounding the ampoule. Annear (1956) reported very good survival of various organisms dried rapidly in vacuo at temperatures which, although unknown, probably avoided drying from the frozen state. More recently, Annear (1958) extended these observations and found very good survival of Vibrio cholerae subjected to fairly rapid vacuum-drying from the liquid state. Annear also found that survival of freeze-dried preparations was generally best when some thawing occurred before drying was complete. As in the experiments of Greiff $e t$ al. the actual temperatures of the specimens were not measured.

There is, therefore, conflicting evidence regarding the drying conditions which best conserve viability. While the reason for the disagreement is not obvious; it is of interest to note that different workers have used a variety of experimental conditions, not all of which are independent. As an example, different suspending fluids obviously produce different chemical environments during drying, but they may also give rise to considerable differences both in the rates of drying and in the steady-state temperatures at which drying proceeds. These latter effects result from differences in rates of diffusion of water and water vapour and also, at times, from differences in thermal properties of the specimens. There is, in fact, a rather complicated interdependence of a number of physical and chemical factors which may conceivably affect the biological result.

The experiments now reported were designed to answer two simple questions: (i) is the mortality during drying greatly affected by whether drying is carried out from the frozen or from the liquid state; (ii) are the effects of the drying temperature dependent on the nature of the solutes present? Although there is no particular novelty in this approach the present experiments permitted the actual temperatures obtained in the specimens to be measured and to be partially controlled during drying. The apparatus used was described by Mellor \& Ohye (1959).

\section{METHODS}

Bacteria were grown in brain + heart infusion broth, aerated by shaking at $30^{\circ}$ for $20 \mathrm{hr}$. Coliphage $\mathbf{T} 2$ was propagated on Escherichia coli B and a lysate in nutrient broth was filtered and stored at $1^{\circ}$. Suspensions of bacteria were prepared by centrifuging a known volume of broth culture and resuspending the organisms in distilled water at $1 / 10$ the original volume. For the factorial experiment, suspensions of Pseudomonas fluorescens prepared in this way were mixed with an equal volume of T 2 lysate. One volume of the mixture was then added to $19 \mathrm{vol}$. of such mixtures of distilled water, mannitol and sucrose solutions as were required to give the desired solute concentrations in the suspensions used for drying. Samples $(0.1 \mathrm{ml}$.) of these suspensions were dispensed in $88 \times 9 \mathrm{~mm}$. ampoules and dried in a vacuum drier described by Mellor \& Ohye (1959). Drying began within 30-60 min. after the organisms were first transferred to the sucrose + mannitol solutions. 
Temperatures of the drying material were measured with 40-gauge thermocouples connected to a recording potentiometer. As the temperatures could be measured concurrently at only 9 points it was not possible to obtain temperature records for each of the $\mathbf{1 6}$ solute combinations used in the factorial experiments. Temperatures were, however, measured for the lowest and highest concentrations of each solute, it being assumed that intermediate solute concentrations would probably have temperature histories within the observed range. During drying the temperature of the material was controlled by regulating the temperature of the copper tube-holder, and by adjusting the total pressure inside the vacuum chamber (Mellor \& Ohye, 1959). During drying at the nominal temperature of $-20^{\circ}$ the total pressure in the dryer was not controlled, consequently in the suspension without added solutes most of the sublimation of ice occurred at a much lower temperature than in those suspensions which contained either or both carbohydrates.

On completion of drying all tubes were removed from the dryer and the contents rehydrated immediately with $1.0 \mathrm{ml}$. nutrient broth. When required further dilutions in nutrient broth were prepared. When mixtures of Pseudomonas fluorescens and coliphage $\mathrm{T} 2$ were being counted, the former organism was plated on brain + heart infusion agar and incubated at $30^{\circ}$ for $48 \mathrm{hr}$. The phage plaques were developed in a layer of soft nutrient agar seeded with Escherichia coli $\mathrm{B}$ and incubated at $37^{\circ}$ for $24 \mathrm{hr}$. Under these conditions $\boldsymbol{P}$. fluorescens did not interfere with normal plaque development.

\section{RESULTS}

The results of several experiments in which micro-organisms were dried at different temperatures showed that the effect of drying temperature on survival depended on the nature of the solutes present in the suspensions. For several organisms, including Salmonella newport, Pseudomonas fluorescens, Vibrio metchnikovi and the coliphages T2 and T5, survival in $0.5 \mathrm{M}$-sucrose was very similar whether drying was carried out at $c .-20^{\circ}$ or $0-2^{\circ}$, but for suspensions in $0.5 \mathrm{M}$-mannitol survival was greatly decreased after drying at the lower temperature. There were also suggestions of differential responses between micro-organisms when the drying conditions were changed. Some of these differences between solutes, drying temperatures and organisms have now been confirmed in an experiment which permitted more decisive comparisons.

In this experiment drying was carried out in solutions of each of the possible 16 combinations of mannitol and sucrose at $0,0 \cdot 25,0.50$ and $0.75 \mathrm{M}$. Survival in each of the 16 solutions was measured after drying at each of three nominal temperatures, namely $-20,0$ and $20^{\circ}$. The actual temperatures for three of the runs are shown in Fig. 1. In order to provide an estimate of error the experiment was carried out twice at each temperature. As it was possible to dry at only one temperature at one time the whole experiment was divided into 6 blocks, the order in which each block was dried being decided by random selection. In this way there was obtained, for each organism, a 
total of 96 estimates of the viable counts after drying, the common logarithms of which have been used for the analyses of variance. By drying together a mixture of two organisms which could be enumerated separately, it was possible to obtain evidence about the survival of both organisms and, at the same time, to ensure that any differences in the responses of the two organisms would not be due to environmental differences during drying and rehydration. The two organisms selected were Pseudomonas fluorescens and coliphage T2.
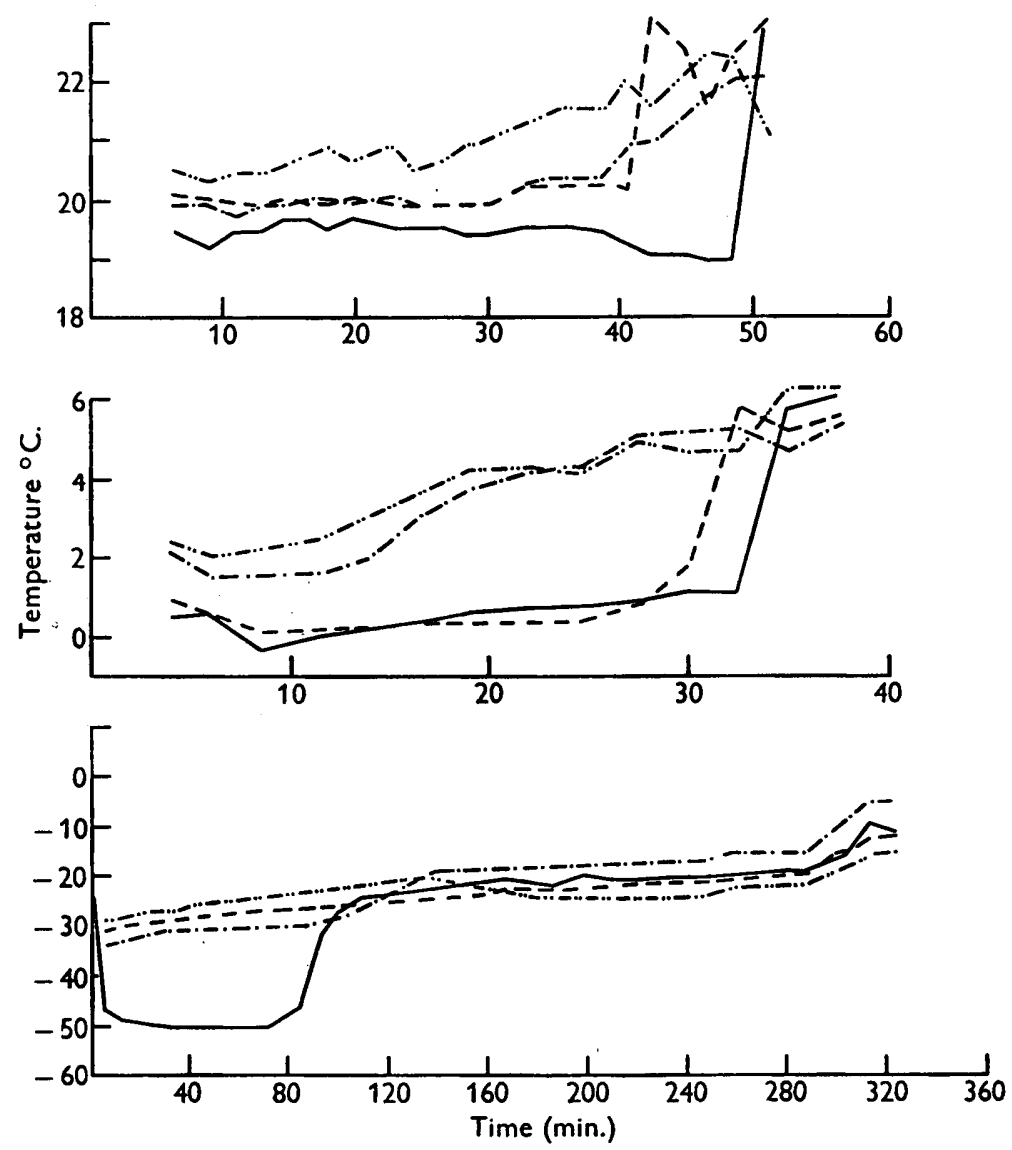

Fig. 1. Temperature changes during drying of coliphage T2 and Pseudomonas fluorescens from suspensions containing different proportions of sucrose and mannitol. no sucrose or mannitol; - - - - mannitol (0.75 M) only; -.-.-., sucrose (0.75 $\mathrm{M})$ only ; -........, sucrose $(0.75 \mathrm{M})$ and mannitol $(0.75 \mathrm{M})$.

A summary of the results is shown in Fig. 2 and Table 2, together with an abridged analysis of variance in Table 1. (Copies of the complete analyses of variance together with a list of the individual results will be supplied to interested persons on request.) For the purposes of the analysis of variance it was assumed that the three nominal drying temperatures were, in fact, equally spaced; this enabled the linear and quadratic components of temperature to 
be examined: Similarly, the equal spacing of the sucrose and mannitol concentrations permitted the separation of the linear, quadratic and cubic components for these factors, and of their interactions with temperature. Five of the 96 samples of coliphage T2 yielded no plaques at the highest dilution plated, indicating a viable population of $<100$ particles $/ \mathrm{ml}$. It was found that when all such counts were assigned the logarithmic value of $2 \cdot 00$, the results would have been almost the same as estimating the missing values. As the principal conclusions appeared to be very clearly established this simpler procedure was adopted.

Table 1. Drying of coliphage $\boldsymbol{T}_{\mathbf{2}}$ and Pseudomonas fluorescens in sucrose and mannitol solutions at each of three temperatures

Analyses of variance of $\log$ viable counts/ml. after drying.

\begin{tabular}{|c|c|c|c|c|c|}
\hline \multirow[b]{2}{*}{ Source of variation } & \multirow{2}{*}{$\begin{array}{l}\text { Degrees } \\
\text { of } \\
\text { freedom }\end{array}$} & \multicolumn{2}{|c|}{ Coliphage $\mathbf{T 2}$} & \multicolumn{2}{|c|}{ Pseudomonas fluorescens } \\
\hline & & Mean square & $\boldsymbol{F}$. & Mean square & $\boldsymbol{F}$ \\
\hline Temperature, $\boldsymbol{T}$ & 2 & $15 \cdot 0998$ & $31 \cdot 34 * *$ & 0.9715 & $1 \cdot 72$ \\
\hline $\begin{array}{l}\text { Linear regression } \\
\text { on } T, T l\end{array}$ & 1 & $29 \cdot 5392$ & $61 \cdot 31 * *$ & 1.9425 & $3 \cdot 44$ \\
\hline $\begin{array}{l}\text { Quadratic regression } \\
\text { on } T, T q\end{array}$ & 1 & 0.6604 & $1 \cdot 37$ & $0 \cdot 0005$ & - \\
\hline Error (whole units) & $\mathbf{3}$ & 0.4818 & - & 0.5639 & - \\
\hline Solutions & 15 & 4.5907 & $8 \cdot 10 * * *$ & $1 \cdot 2324$ & $6 \cdot 4,1 * * *$ \\
\hline Sucrose $\boldsymbol{S}$ & $\mathbf{3}$ & 0.9388 & $1 \cdot 66$ & $5 \cdot 8362$ & $30 \cdot 35^{* * *}$ \\
\hline Mannitol $\boldsymbol{M}$ & 3 & $20 \cdot 4509$ & $36 \cdot 08 * * *$ & 0.0668 & - \\
\hline Interaction $\boldsymbol{S} \times \boldsymbol{M}$ & 9 & $0: 5212$ & - & 0.0864 & - \\
\hline Solutions $\times T$ & 30 & 4.5727 & $8 \cdot 07 * * *$ & 0.7127 & $3 \cdot 71 * * *$ \\
\hline$T l \times S$ & 3 & $26 \cdot 2996$ & $46 \cdot 40^{* * *}$ & $2 \cdot 8369$ & $14 \cdot 75^{* * *}$ \\
\hline$T l \times M$ & 3 & $\mathbf{2 \cdot 2 3 8 9}$ & $\mathbf{3 \cdot 9 5 *}$ & 0.5076 & $2 \cdot 64$ \\
\hline$T q \times S$ & 3 & $2 \cdot 5081$ & $4 \cdot 43 * *$ & 0.2378 & $\mathbf{1} \cdot \mathbf{2 4}$ \\
\hline$T q \times M$ & 3 & $4 \cdot 3513$ & $7 \cdot 68 * * *$ & 0.5328 & $2 \cdot 77$ \\
\hline$T l \times S \times M$ & 9 & $1 \cdot 0523$ & $1 \cdot 86$ & 0.5354 & $2 \cdot 78^{*}$ \\
\hline$T q \times S \times M$ & 9 & $2 \cdot 3908$ & $4 \cdot 22 * * *$ & 0.4686 & $2 \cdot 44 \neq$ \\
\hline Error (sub units) & 45 & 0.5668 & - & $0 \cdot 1923$ & - \\
\hline
\end{tabular}

$*$, **, *** signifies $P<0.05,<0.01,<0.001$ respectively.

From Table 1 it may be seen that there is a large average effect of temperature for coliphage $\mathrm{T} 2$, which is almost completely accounted for by the linear regression. For Pseudomonas fluorescens, on the other hand, the average effect of temperature is not significant. Table 2 and Fig. 2 show that the average survival of coliphage $\mathrm{T} 2$ was increased as the drying temperature was changed from $-20^{\circ}$ to $20^{\circ}$. Also from Table 1 it may be seen that there were significant average effects of solutions for each organism, the beneficial effect of mannitol being largely responsible in the case of coliphage T2, and of sucrose in the case of $\boldsymbol{P}$. fluorescens. Consideration of only the average effects, however, is not justified since, for both organisms, the interactions : between solutions and temperature are very highly significant.

The large effects of mannitol on the survival of coliphage T2 are mainly linear, being roughly proportional to the concentration (Table 2 ). Though there is no significant average effect of sucrose for coliphage $\mathrm{T} 2$, there is a very 
large interaction between temperature and sucrose (Tables 1 and 2), drying at $20^{\circ}$ giving much better survival at the lowest two concentrations of sucrose $\left(0\right.$ and $0.25 \mathrm{M}$ ), and $-20^{\circ}$ giving better results with the highest two concentrations. Sucrose increased the survival after drying at $-20^{\circ}$ by about 1000 -fold, whereas at 0 or $20^{\circ}$. its effect was a decrease of 10 -fold or 250-fold, respectively. The interpretation of the effects of solutions on coliphage $\mathbf{T} 2$ is complicated by the number of significant interactions, especially the highly significant second-order interactions $(T q \times S \times M)$. Table 2 shows that mannitol increased survival of coliphage $\mathrm{T} 2$ at all three drying temperatures; drying at $-20^{\circ}$ gave the smallest survival at all concentrations of mannitol. Figure 2. shows that at $20^{\circ}$, which gave the best average survival of coliphage T2, mannitol had little effect at the lowest two concentrations of sucrose, but was beneficial at the highest two concentrations. Sucrose was deleterious at all concentrations of mannitol for coliphage $\mathrm{T} 2$ at $20^{\circ}$, but at $0^{\circ}$ it was detrimental only at the higher concentrations and was beneficial in the absence of mannitol. At $-20^{\circ}$, sucrose was beneficial at all concentrations of mannitol, while mannitol was. beneficial at all concentrations of sucrose.

Table 2. Solute temperature interactions for coliphage T2 and Pseudomonas fluorescens dried at each of three temperatures

\begin{tabular}{|c|c|c|c|c|c|c|c|c|c|}
\hline \multirow[b]{2}{*}{ Solute } & \multirow{2}{*}{$\begin{array}{c}\text { Concn. } \\
\text { (M) }\end{array}$} & \multicolumn{4}{|c|}{ Coliphage T2 } & \multicolumn{4}{|c|}{ P. fluorescens } \\
\hline & & \multicolumn{8}{|c|}{ log viable count/ml. } \\
\hline Sucrose & $\begin{array}{l}0.0 \\
0.25 \\
0.50 \\
0.75\end{array}$ & $\begin{array}{l}2 \cdot 69 \dagger \\
4 \cdot 96 \\
5 \cdot 93 \\
5 \cdot 56\end{array}$ & $\begin{array}{l}\mathbf{6 \cdot 2 7} \\
\mathbf{5 \cdot 5 6} \\
5 \cdot 43 \\
5 \cdot 30\end{array}$ & $\begin{array}{l}7 \cdot 48 \\
6 \cdot 80 \\
5 \cdot 26 \\
5 \cdot 02\end{array}$ & $\begin{array}{l}\mathbf{5 \cdot 4 8} \\
\mathbf{5} \cdot 77 \\
\mathbf{5 \cdot 5 4} \\
\mathbf{5 \cdot 2 9}\end{array}$ & $\begin{array}{l}7 \cdot 02 \\
8 \cdot 91 \\
8 \cdot 97 \\
9 \cdot 04\end{array}$ & $\begin{array}{l}8 \cdot 13 \\
8 \cdot 78 \\
8 \cdot 90 \\
8 \cdot 84\end{array}$ & $\begin{array}{l}8 \cdot 62 \\
9 \cdot 00 \\
8 \cdot 93 \\
8 \cdot 79\end{array}$ & $\begin{array}{l}7 \cdot 92 \\
8 \cdot 89 \\
8 \cdot 94 \\
8 \cdot 89\end{array}$ \\
\hline $\begin{array}{l}\text { Sucrose } \\
\text { and } \\
\text { mannitol }\end{array}$ & Mean & $4-78$ & $5 \cdot 64$ & $6 \cdot 14$ & $\mathbf{5 \cdot 5 2}$ & $8 \cdot 48$ & $8 \cdot 66$ & $8 \cdot 83$ & $8 \cdot 66$ \\
\hline Mannitol & $\begin{array}{l}0.0 \\
0.25 \\
0.50 \\
0.75\end{array}$ & $\begin{array}{l}\mathbf{3} \cdot 33 \\
\mathbf{5} \cdot 28 \\
\mathbf{5} \cdot 10 \\
\mathbf{5 \cdot 4 2}\end{array}$ & $\begin{array}{l}3 \cdot 61 \\
5 \cdot 49 \\
6 \cdot 36 \\
7 \cdot 10\end{array}$ & $\begin{array}{l}5 \cdot 76 \\
6 \cdot 01 \\
6 \cdot 10 \\
6 \cdot 70\end{array}$ & $\begin{array}{l}4 \cdot 23 \\
5 \cdot 59 \\
5 \cdot 86 \\
6 \cdot 40\end{array}$ & $\begin{array}{l}8 \cdot 88 \\
8 \cdot 22 \\
8 \cdot 55 \\
8 \cdot 28\end{array}$ & $\begin{array}{l}8 \cdot 39 \\
8 \cdot 65 \\
8 \cdot 76 \\
8 \cdot 86\end{array}$ & $\begin{array}{l}8 \cdot 80 \\
8 \cdot 87 \\
8 \cdot 75 \\
8 \cdot 91\end{array}$ & $\begin{array}{l}8 \cdot 60 \\
8 \cdot 58 \\
8 \cdot 68 \\
8 \cdot 60\end{array}$ \\
\hline \multicolumn{2}{|c|}{ Before drying } & \multicolumn{4}{|c|}{$8 \cdot 46 \ddagger$} & \multicolumn{4}{|c|}{$9 \cdot 12 \ddagger$} \\
\hline
\end{tabular}

$\dagger$ Entries at each temperature and solute concentration are the logarithmic mean of 8 values.

$\ddagger$ Logarithmic mean of 24 values.

For Pseudomonas fluorescens, the effects of sucrose were much smaller than for coliphage T2 even though its average effect was significant for the bacterium (Table 1). The influence of a temperature-sucrose interaction (Table 1) can be seen in the fact that, whereas the absence of sucrose caused the survival of $\boldsymbol{P}$. fluorescens to increase by 40 -fold as the drying temperature was increased from -20 to $20^{\circ}$, the presence of sucrose caused the effect of temperature to disappear. Alternatively stated, sucrose solutions increased survival of this organism, dried at $-20^{\circ}, 0^{\circ}$ and $20^{\circ}$, by factors of $c .100,6$ and 2 , respectively (Table 2). Although the temperature-mannitol interactions shown in Table 1 
are not significant for $\boldsymbol{P}$. fluorescens, it may be pointed out that the two second-order interactions are significant, as are two of the subdivided interactions $(T l \times M c ; T q \times M c)$ which are not tabulated. The tendency for the effects of mannitol on $\boldsymbol{P}$. fluorescens to be most marked in the absence of sucrose, and to depend also on the temperature (Fig. 2; Table 2) may therefore be real.
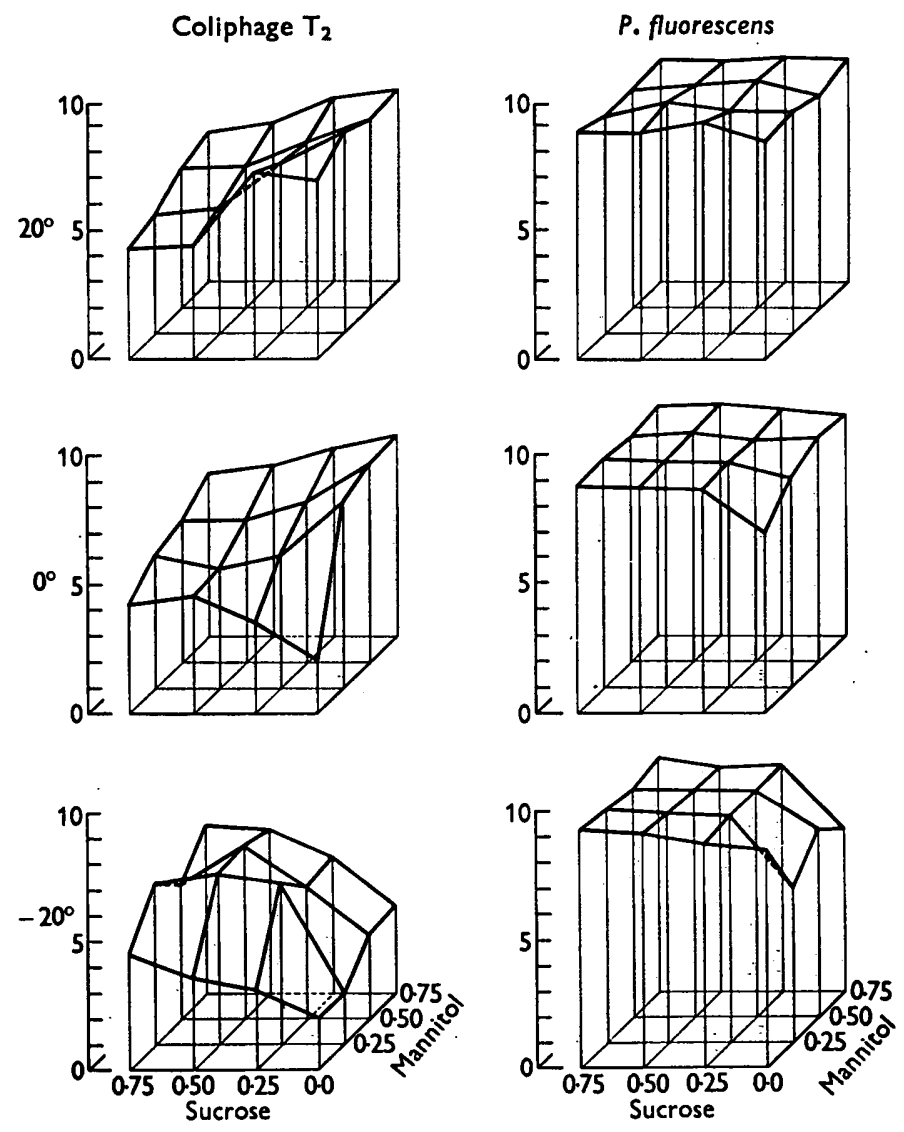

Fig. 2. Solute temperature interactions for coliphage T2 and Pseudomonas fluorescens dried in 16 sucrose and/or mannitol solutions at each of three temperatures. Heights of the vertical lines represent the logs of the viable populations/ml. immediately after drying. The scale is shown on the left of each diagram. The concentrations of solutes are given as molarities.

When all drying temperatures are considered (Fig. 2), the best survivals of coliphage $\mathrm{T} 2$, which were just over $30 \%$ of the pre-drying counts, were obtained by drying at $0^{\circ}$ or $20^{\circ}$ in the absence of sucrose and in the presence of mannitol. For Pseudomonas fluorescens, several treatments gave survivals which were close to $100 \%$ of the numbers present before drying. 


\section{DISCUSSION}

In part, the present results support those of some earlier workers who obtained superior survival by drying from the liquid state (Stamp, 1947; Mazur \& Weston, 1956; Annear, 1958). In part, they also support the results of those who have reported that drying from the frozen state is either necessary or desirable (Proom \& Hemmons, 1949; Hutton, Hilmoe \& Roberts, 1951; Fry \& Greaves, 1951). The results can also be used to show that sucrose and mannitol do not necessarily produce similar effects, and that either substance may be beneficial or damaging according to the conditions and the test organism. Taken as a whole, however, the results show that the choice of drying temperature depends on the nature of the solutes present. As the results do not support any simple hypothesis about the drying temperature or combinations of sucrose and mannitol which result in the best retention of viability, it is concluded that some additional factors are probably decisive. The nature of the additional factors is, of course, not revealed by the present experiments.

The authors are greatly indebted to Mr G. G. Coote, C.S.I.R.O. Division of Mathe matical Statistics, for the analyses of variance.

\section{REFERENCES}

Annear, D. I. (1956). The preservation of bacteria by drying in peptone plugs. J. Hyg., Camb. 54, 487.

Annear, D. I. (1958). Observations on drying bacteria from the frozen and from the liquid state. Austr. J. exp. Biol. 36, 211.

FRY, R. M. (1954). The preservation of bacteria. Biological Applications of Freezing and Drying, p. 215. New York: Academic Press.

Fry, R. M. \& Greaves, R. I. N. (1951). The survival of bacteria during and after drying. J. Hyg., Camb. 49, 220.

Greiff, D., Blumenthal, H., Chiga, M. \& Pinkerton, H. (1954). The effects on biological materials of freezing and drying by vacuum sublimation. II. Effect on influenza virus. J. exp. Med. 100, 89.

Harris, R. J. C. (1954). The preservation of viruses. Biological Applications of Freezing and Drying, p. 201. New York: Academic Press.

Hutton, R. S., Hilmoe, R. J. \& Roberts, J. L. (1951). Some physical factors that influence the survival of Brucella abortus during freeze-drying. J. Bact. 61, 309.

Mazur, P. \& Weston, W. H. (1956). The effects of spray-drying on the viability of fungus spores. $J$. Bact. 71, 257.

Mellor, J. D. \& ОhYe, D. F. (1959). An ampoule freeze-dryer for microbiological research. Vacuum 9 , nos. 5 \& 6.

Proom, H. \& Hemmons, L. M. (1949). The drying and preservation of bacterial cultures. J. gen. Microbiol. $3, \dot{7}$.

Stamp, LoRd (1947). The preservation of bacteria by drying. J. gen. Microbiol. 1, 251.

(Received 4 June 1959) 